\title{
Final Technial Report Stochastic Nonlinear Data-Reduction Methods with Detection and Prediction of Critical Rare Event DE-SC0002542 (ER25923)
}

\author{
PI: George Em Karniadakis \\ Division of Applied Mathematics, Brown University
}

Collaborators at Other Institutes: Eric Vanden-Eijnden, Courant Institute, New York University; Guang Lin, Pacific Northwest National Laboratory and Xiaoliang Wan, Louisiana State University

Dates of Performance: 09/15/09 - 09/15/12

\section{Objectives of this project}

In this project, the collective efforts of all co-PIs aim to address three current limitations in modeling stochastic systems: (1) the inputs are mostly based on ad hoc models, (2) the number of independent parameters is very high, and (3) rare and critical events are difficult to capture with existing algorithms.

To overcome these problems, we propose research on the following three topics: (1) development of certified low-dimensional models for effective reduction of dimensionality, (2) development of a scalable sensitivity-based hierarchical uncertainty quantification approach, and (3) development of algorithms for real-time anomaly detection and rare events prediction.

The Brown PI has focused primarily on topic (1). The main idea is to formulate new petabyte data-reduction techniques based on fundamental extensions of the proper orthogonal decomposition (POD) to include nonlinearity and stochasticity. Here in the final report we summarize and compare two very promising low-dimensional methods for time evolving systems, which they can be thought of as a combination of POD and polynomial chaos as they have the best features of both. The mathematical formulations are slightly different but equivalent as we discuss next.

A new approach, called Dynamically Orthogonal (DO) method, was developed in [1]; the idea is to represent the solution in a more general expansion, i.e.,

$$
u(x, t ; \omega)=\bar{u}(x, t)+\sum_{i=1}^{N} Y_{i}(t ; \omega) u_{i}(x, t),
$$


where $Y_{i}(t ; \omega)$ are stochastic processes, $u_{i}(x, t)$ orthonormal fields and $\bar{u}(x, t)$ is the mean. The time dependence on both the stochastic coefficients and the basis fields makes the above representation very flexible for the representation of strongly transient, non-stationary responses. However, this same property makes the representation redundant and the derivation of well-posed equations for all the quantities involved is not a straightforward problem. In [1] it was illustrated how this redundancy can be overcome by adopting a natural constraint: the dynamical orthogonality condition. It was shown that using this condition a set of evolution equations for the $Y_{i}(t ; \omega), u_{i}(x, t)$ and $\bar{u}(x, t)$ can be derived. These derived field equations are consistent with existing methods such as proper orthogonal decomposition method (POD) and PC.

In addition, we formulate an alternative approach, Bi-Orthogonal (BO) method [2]; the idea is the same as DO where we have time-dependence on both the stochastic coefficients and the basis fields. The difference between DO and BO is that we impose in BO both the stochastic coefficients and the basis to be orthogonal in time while, in DO, only the basis maintains the orthogonality in time. We derive the corresponding $\mathrm{BO}$ evolution equations in which the inner product of the basis (or the stochastic coefficients) and its time derivatives under the underlying measure can be explicitly expressed in terms of the differential operator, the basis and the stochastic coefficients. We also demonstrate that $\mathrm{DO}$ and $\mathrm{BO}$ are equivalent through the matrix ordinary differential equation that gives an one-to-one mapping between $\mathrm{BO}$ and $\mathrm{DO}$ components. BO can replace DO in the aforementioned hybrid method to overcome the singularity.

\section{An overview of the DO equations and a hybrid DO-PC method}

We consider the following SPDE:

$$
\begin{aligned}
\frac{\partial u}{\partial t} & =\mathcal{L}(u(t, x ; \omega)), \quad x \in D, \omega \in \Omega \\
u\left(t_{0}, x ; \omega\right) & =u_{0}(x ; \omega), \quad x \in D, \omega \in \Omega \\
\mathcal{B}[u(t, x ; \omega)] & =h(t, x ; \omega), \quad x \in \partial D, \omega \in \Omega,
\end{aligned}
$$

where $\mathcal{L}$ is a differential operator and $\mathcal{B}$ is a linear differential operator. $D$ is a bounded domain in $\mathcal{R}^{d}$ where $d=1,2$, or 3 .

\subsection{Definitions}

Let $(\Omega, \mathcal{F}, P)$ be a probability space, where $\Omega$ is the sample space, $\mathcal{F}$ is the $\sigma$-algebra of subsets of $\Omega$, and $P$ is a probability measure. For a random field $u(x, t ; \omega), \omega \in \Omega$, the expectation operator of $u$ is defined as

$$
\bar{u}(x, t)=E[u(x, t ; \omega)]=\int_{\Omega} u(x, t ; \omega) d P(\omega) .
$$

The set of all continuous and square integrable random fields , i.e., $\int_{D} E\left[u(x, t ; \omega)^{T} u(x, t ; \omega)\right] d x<$ $\infty$, where $u(x, t ; \omega)^{T}$ is the transpose of $u$, for all $t \in T$ and the bi-linear form of the covariance 
operator

$$
C_{u(\cdot, t ; \omega) v(\cdot, s ; \omega)}(x, y)=E\left[(u(x, t ; \omega)-\bar{u}(x, t))^{\prime}(v(x, s ; \omega)-\bar{v}(x, s))\right], \quad x, y \in D,
$$

form a Hilbert space that will be denoted by $\mathcal{H}[3,4]$. For $u(x, t ; \omega), v(x, t ; \omega) \in \mathcal{H}$, the spatial inner product is defined as

$$
<u(\cdot, t ; \omega), v(\cdot, t ; \omega)>=\int_{D} u(x, t ; \omega)^{T} v(x, t ; \omega) d x .
$$

We define the projection operator $\Phi_{S}$ of a field $u(x, t), x \in D$ to an $m$-dimensional linear subspace $S$ spanned by the orthogonal basis $S=\left\{w_{i}(x, t ; \omega)\right\}_{i=1}^{m}, x \in D$ as follows:

$$
\Phi_{S}[u(x, t ; \omega)]=\sum_{i=1}^{m}<w_{i}(\cdot, t ; \omega), u(\cdot, t ; \omega)>w_{i}(x, t ; \omega)
$$

\subsection{DO representation}

Using a time-dependent generalization of the Karhunen-Loeve (KL) expansion [1], we have that every random field $u(x, t ; \omega) \in \mathcal{H}$ at a given time $t$ can be approximated by a finite series of the form

$$
u(x, t ; \omega)=\bar{u}(x, t)+\sum_{i=1}^{N} Y_{i}(t ; \omega) u_{i}(x, t),
$$

where $u_{i}(x, t)$ are the eigenfunctions, and $Y_{i}(t ; \omega)$ are zero-mean stochastic processes whose variance $E\left[Y^{2}(t ; \omega)\right]$ is equal to the corresponding eigenvalue $\lambda_{i}(t)$ of the eigenvalue problem of the KarhunenLoeve decomposition:

$$
\int_{D} C_{u(\cdot, t) u(\cdot, t)}(x, y) u_{i}(x, t) d x=\lambda_{i}(t) u_{i}(y, t), \quad y \in D .
$$

We define the linear subspace $V_{S}=\operatorname{span}\left\{u_{i}(x, t)\right\}_{i=1}^{N}$ as the linear space spanned by the $N$ deterministic eigenfields associated with the $N$ largest eigenvalues. Note that both the stochastic coefficients $Y_{i}(t ; \omega)$ and the orthogonal basis $u_{i}(x, t)$ are time-dependent (and they are evolving according to the system dynamics) unlike other methods such as the standard PC where the stochastic coefficients are time-independent. In [5], a similar expansion with time evolving PC basis is presented but the time-depended basis is obtained according to the PDF of the solution; in DO it is obtained through an evolution equation as we explain next.

\subsection{DO field equations}

All quantities $\bar{u}(x, t), u_{i}(x, t), Y_{i}(t ; \omega), i=1, \ldots, N$ in the representation (2) are time-dependent and hence there exists some redundancy in the representation. Therefore, additional constraints need 
to be imposed in order to formulate a well posed problem for the unknown quantities. As first proposed in [1], a natural constraint to overcome redundancy is that the evolution of the basis $\left\{u_{i}(x, t)\right\}_{i=1}^{N}$ be normal to the space $V_{S}$; this can be expressed through the following condition:

$$
\frac{d V_{S}}{d t} \perp V_{S} \Leftrightarrow\left\langle\frac{\partial u_{i}(x, t)}{\partial t}, u_{j}(x, t)\right\rangle=0 \quad i, j=1, \ldots, N
$$

This condition is referred to as the dynamically orthogonal (DO) condition. Note that the DO condition preserves orthonormality of the basis $\left\{u_{i}(x, t)\right\}_{i=1}^{N}$ since

$$
\frac{\partial}{\partial t}<u_{i}(\cdot, t), u_{j}(\cdot, t)>=<\frac{\partial u_{i}(\cdot, t)}{\partial t}, u_{j}(\cdot, t)>+<u_{i}(\cdot, t), \frac{\partial u_{j}(\cdot, t)}{\partial t}>=0, \quad i, j=1, \ldots, N .
$$

It is proved in [1] that the DO condition leads to a set of independent and explicit evolution equations for all the unknown quantities. Next, we state the DO evolution equations without proof:

Theorem 1. Under the assumptions of the DO representation, the original SPDE (1a)-(1c) is reduced to the following system of equations

$$
\begin{aligned}
\frac{\partial \bar{u}(t, x)}{\partial t} & =E[\mathcal{L}[u(\cdot, t ; \omega)]], \\
\frac{d Y_{i}(t ; \omega)}{d t} & =<\mathcal{L}[u(\cdot, t ; \omega)]-E[\mathcal{L}[u(\cdot, t ; \omega)]], u_{i}(\cdot, t)>, \quad i=1, \ldots, N \\
\sum_{i=1}^{N} C_{Y_{i}(t) Y_{j}(t)} \frac{\partial u_{i}(t, x)}{\partial t} & =\prod_{V_{s}^{\perp}} E\left[\mathcal{L}[u(\cdot, t ; \omega)] Y_{j}\right], \quad j=1, \ldots, N,
\end{aligned}
$$

where the projection in the orthogonal complement of the linear subspace is defined as $\prod_{V_{S}^{\perp}} F(x)=$ $F(x)-\prod_{V_{S}} F(x)=F(x)-\sum_{k=1}^{N}<F(\cdot), u_{k}(\cdot, t)>u_{k}(\cdot, t)$ and the covariance of the stochastic coefficients is $C_{Y_{i}(t) Y_{j}(t)}=E\left[Y_{i}(t ; \omega) Y_{j}(t ; \omega)\right]$. The associated boundary conditions have the form

$$
\begin{aligned}
\left.\mathcal{B}[\bar{u}(\xi, t ; \omega)]\right|_{\xi \in \partial D} & =E[h(\xi, t ; \omega)], \\
\left.\mathcal{B}\left[u_{i}(\xi, t)\right]\right|_{\xi \in \partial D} & =E\left[Y_{j}(t ; \omega) h(\xi, t ; \omega)\right] C_{Y_{i}(t) Y_{j}(t)}^{-1},
\end{aligned}
$$

and the initial conditions for the DO components are given by

$$
\begin{aligned}
\bar{u}\left(x, t_{0}\right) & =E\left[u_{0}(x ; \omega)\right], \\
Y_{i}\left(t_{0} ; \omega\right) & =<u_{0}(\cdot, \omega)-\bar{u}\left(x, t_{0}\right), v_{i}(\cdot)>, \\
u_{i}\left(x, t_{0}\right) & =v_{i}(x),
\end{aligned}
$$

for all $i=1, \ldots, n$, where $v_{i}(x)$ are the eigenfields of the covariance operator $C_{u\left(\cdot, t_{0}\right) u\left(\cdot, t_{0}\right)}$ defined by the following eigenvalue problem for $t=t_{0}$ :

$$
\int_{D} C_{u\left(\cdot, t_{0}\right) u\left(\cdot, t_{0}\right)}(x, y) v_{i}(x) d x=\lambda_{i}(t) v_{i}(y), \quad y \in D .
$$


Remark 1. The DO evolution equations (5a)-(5c) are derived by using the DO conditions and DO representation. It is shown in [1] that by imposing suitable restrictions on the DO representation the equations for methods such as Polynomial Chaos or Proper Orthogonal Decomposition (POD) can be recovered from the DO evolution equations. For example, $P C$ can be recovered by setting $Y_{i}(t ; \omega)=\Psi_{i}(\xi(\omega))$, where $\Psi_{i}(\xi)$ is an orthogonal polynomial in terms of $\xi$.

Remark 2. From the DO representation, the moments can be readily computed. For example, the first moment, i.e., the mean, appears in the DO representation as $\bar{u}(x, t)$ while the second moment is directly computed as follows:

$$
\operatorname{Var}[u]=E\left[(u-\bar{u})^{2}\right]=E\left[\left(\sum_{i=1}^{N} u_{i} Y_{i}\right)^{2}\right]=\sum_{i, j=1}^{N} u_{i}(x, t) E\left[Y_{i} Y_{j}\right] u_{j}(x, t) .
$$

As the DO representation at any fixed time $t$ can be seen as Karhunen-Loeve decomposition, there is a relationship between the eigenpairs for the covariance matrix of $Y_{i}(t ; \omega), i=1, \ldots, N$ and the eigenpairs for the covariance operator of $u(x, t ; \omega)$. For the covariance matrix $C$ whose $(i, j)$-th element is $C_{i j}=C_{Y_{i}(t) Y_{j}(t)}$, we have a set of eigenvalues and eigenvectors that satisfies the following eigenvalue problem

$$
C(t) \phi_{k}(t)=\rho_{k} \phi_{k}(t), k=1, \ldots, N
$$

where $\phi_{k}(t)=\left(\phi_{k 1}(t), \cdots, \phi_{k N}(t)\right)^{T}$. Similarly, for the covariance operator for $u(x, t ; \omega)$, there exists a set of eigenvalues and eigenfields for $C_{u}(x, y)$ through the Karhunen-Loeve decomposition such that

$$
\int_{D} C_{u}(x, y) v_{k}(x, t) d x=\lambda_{k} v_{k}(y, t)
$$

where $C_{u}(x, y)=E[(u(x, t ; \omega)-\bar{u}(x, t))(u(y, t ; \omega)-\bar{u}(y, t))]$. In order to relate the eigenvalues and eigenvectors for $Y_{i}$ with those for $u(x, t ; \omega)$, we substitute the DO representation of $u$ into $C_{u}(x, y)$ and compare Equations (7) and (8) to obtain the following relations:

$$
\lambda_{k}=\rho_{k} ; \quad v_{k}(x, t)=\phi_{k l}(t) u_{l}(x, t) .
$$

This shows that the stochastic coefficients $Y_{i}$ together with the modes $u_{i}$ provide the necessary information to describe both the shape and magnitude of the uncertainty that characterizes a stochastic field but also the principal directions in $\mathcal{H}$ over which this stochasticity is distributed.

\subsection{Hybrid method: combining PC with DO}

In Theorem 1, it is assumed that the initial condition for the SPDE is random from which the corresponding initial conditions for DO components are derived. However, in practice in many cases the initial condition for the SPDE is deterministic while the randomness comes from other sources such as random coefficients or random forcing. Then $Y_{i}, i=1, \ldots, N$ at the initial time 
becomes zero, which makes the covariance matrix for $Y_{i}$ singular. Although the singular limit for the DO equations exist, the transition to finite covariance creates numerical issues. Most importantly, in such a case it is not clear what is the optimal choice to initiate the stochastic subspace. For such problems we propose a hybrid approach of Polynomial Chaos (PC) and DO methods in order to avoid the aforementioned problems. Specifically, for PC we employ the probabilistic collocation method (PCM) or multi-element PCM (ME-PCM), which was found to effectively deal with problems exhibiting low regularity in parametric space as well as for long-term integration [6]. We first use PCM or ME-PCM from the initial time $t_{0}$ up to some time, say $t_{s}$, provided that the stochasticity is sufficiently developed, and then switch over to the DO method at $t_{s}$ and employ the KL decomposition to initialize $\bar{u},\left\{Y_{i}\right\}$ and $\left\{u_{i}\right\}$.

First, we construct the covariance matrix $C_{u\left(\cdot, t_{s}\right)}(x, y)$

$$
C_{u\left(\cdot, t_{s}\right)}(x, y)=E\left[\left(u\left(x, t_{s}\right)-\bar{u}\left(x, t_{s}\right)\right)\left(u\left(y, t_{s}\right)-\bar{u}\left(y, t_{s}\right)\right)\right],
$$

where $u$ and $\bar{u}$ at $t=t_{s}$ are known from PC computations. Then, we compute the eigenpairs for $C_{u\left(\cdot, t_{s}\right)}(x, y)$ by solving

$$
\int_{D} C_{u\left(\cdot, t_{s}\right)}(x, y) \phi(x) d x=\lambda \phi(y)
$$

By setting

$$
u_{i}\left(x, t_{s}\right)=\frac{\phi_{i}(x)}{\left\|\phi_{i}\right\|} \text { and } Y_{i}\left(t_{s}, \omega\right)=<u\left(x, t_{s} ; \omega\right)-\bar{u}(x, t), u_{i}>
$$

we initialize the DO components at $t=t_{s}$ and we are ready to solve the DO evolution equations. This procedure is summarized in Algorithm 1.

Algorithm 1 Hybrid approach of PC and DO method

1: Run PCM or ME-PCM up to $t=t_{s}$ from $t=0$.

2: At $t=t_{s}$, use the KL decomposition for the solution:

$$
u\left(x, t_{s} ; \omega\right)=\bar{u}\left(x, t_{s}\right)+\sum_{i=1}^{N} Y_{i}\left(t_{s} ; \omega\right) \phi_{i}\left(x, t_{s}\right) .
$$

From the KL decomposition, we can initialize $\bar{u}\left(x, t_{s}\right),\left\{Y_{i}\left(t_{s} ; \omega\right)\right\}$ and $\left\{u_{i}\left(x, t_{s}\right)\right\}$ for DO method. 3: Switch over to the DO method up to time $t=t_{f}$.

We will illustrate how the DO evolution equations are used for solving two SPDEs: (i) advection equation in this section and (ii) Burgers equation in the next section. Both are assumed to have deterministic initial conditions to illustrate the advantages of the proposed hybrid approach. 


\section{Bi-Orthogonal condition and field equations}

We introduce a slightly different representation from DO and present corresponding evolution equations [2]. The KL expansion [1] is bi-orthogonal since the stochastic coefficients $Y_{i}$ are orthogonal in the probability space while the eigenfunctions $u_{i}$ are orthogonal in the spatial space. This gives the best approximation to the original process in the sense that it minimizes the total mean-square error. We introduce the bi-orthogonal representation (BO) that has the same form as DO but assumes bi-orthogonality of the stochastic coefficients and the basis in time, instead of DO conditions, and derive its evolution equations. In other words, we have the following conditions:

$$
<u_{i}(\cdot, t), u_{j}(\cdot, t)>=\lambda_{i}(t) \delta_{i j}, \quad E\left[Y_{i} Y_{j}\right](t)=\delta_{i j}, \quad i, j=1, \ldots, N,
$$

where $\left\{\lambda_{i}, u_{i}\right\}$ is the eigenpair of the covariance operator in the KL decomposition. This condition is referred to as the bi-orthogonal (BO) condition. Note the difference between the DO and BO condition; the basis in the DO condition evolves normal to the space $V_{s}$, which maintains the basis to be orthogonal in time, while both the basis and the stochastic coefficients in the $\mathrm{BO}$ condition are orthogonal in time in the associated space, respectively. There is a slight difference between the DO and BO representation; the stochastic coefficients carry the eigenvalue of the covariance operator in the DO representation while the basis carry the eigenvalue of the covariance operator in the $\mathrm{BO}$ representation.

Remark 3. Both the basis and the stochastic coefficients change in time while maintaining the orthogonality. Define the matrix $S$ and $M$ whose entries are

$$
\begin{aligned}
S_{i j} & =\left\langle u_{i}, \frac{\partial u_{j}}{\partial t}\right\rangle, \\
M_{i j} & =E\left[Y_{i} \frac{d Y_{j}}{d t}\right] .
\end{aligned}
$$

Then, by taking derivative for the first term in equation (9) with respect to time, we have $<\frac{\partial u_{i}}{\partial t}, u_{j}>$ $+<u_{i}, \frac{\partial u_{j}}{\partial t}>=0$ for $i \neq j$ and $<\frac{\partial u_{i}}{\partial t}, u_{i}>=\frac{1}{2} \frac{d \lambda_{i}(t)}{d t}$ for $i=j$ or $S_{i j}=-S_{j i}$ for $i \neq j$ and $S_{i i}=\frac{1}{2} \frac{d \lambda_{i}(t)}{d t}$. Similarly, we have $M_{i j}=-M_{j i}$ for $i \neq j$ and $M_{i i}=0$. Note that $M$ is skewsymmetric. It will be shown later that the matrices $S$ and $M$, i.e. the rate of how the basis and the stochastic coefficients change in time, have explicit form.

Next we formulate the BO evolution equations.

Theorem 2. Under the assumptions of the BO representation, the original SPDE (1a)-(1c) is 
reduced to the following system of equations

$$
\begin{aligned}
\frac{\partial \bar{u}(t, x)}{\partial t} & =E[\mathcal{L}[u]], \\
\lambda_{j} \frac{d Y_{j}(t ; \omega)}{d t} & =-\sum_{i=1}^{N} S_{j i} Y_{i}+h_{j}, \quad j=1, \ldots, N, \\
\frac{\partial u_{j}(t, x)}{\partial t} & =-\sum_{i=1}^{N} M_{j i} u_{i}+p_{j}, \quad j=1, \ldots, N,
\end{aligned}
$$

where the entries for the matrix $G, M$ and $S$ and the vectors $\mathbf{h}$ and $\mathbf{p}$ are given as follows:

$$
\begin{aligned}
G_{i j} & =<E\left[\mathcal{L}[u] Y_{j}\right], u_{i}> \\
M_{i j} & = \begin{cases}\frac{G_{i j}+G_{j i}}{-\lambda_{i}+\lambda_{j}}, & \text { if } i \neq j \\
0, & \text { if } i=j\end{cases} \\
S_{i j} & = \begin{cases}G_{i j}+\lambda_{i} M_{i j}, & \text { if } i \neq j \\
G_{i i}, & \text { if } i=j\end{cases} \\
h_{j} & =<\mathcal{L}[u]-E[\mathcal{L}[u]], u_{j}(\cdot, t)> \\
p_{j} & =E\left[\mathcal{L}[u] Y_{j}\right] .
\end{aligned}
$$

Proof. First we insert the BO representation to the $\operatorname{SPDE}(1 \mathrm{a})$ to obtain

$$
\frac{\partial \bar{u}}{\partial t}+\sum_{i=1}^{N} \frac{d Y_{i}}{d t} u_{i}+\sum_{i=1}^{N} Y_{i} \frac{\partial u_{i}}{\partial t}=\mathcal{L}[u]
$$

By applying the mean value operator we obtain the first equation of the theorem (12a). By taking the inner product of the evolution equation (14) with each of the fields $\left\{u_{j}(x, t)\right\}_{j=1}^{N}$ we have

$$
<\frac{\partial \bar{u}}{\partial t}, u_{j}>+\sum_{i=1}^{N}<u_{i}, u_{j}>\frac{d Y_{i}}{d t}+\sum_{i=1}^{N} Y_{i}<\frac{\partial u_{i}}{\partial t}, u_{j}>=<\mathcal{L}[u], u_{j}>
$$

Now, we define the matrix $S$ that has the entries $S_{i j}=<u_{i}, \frac{\partial u_{j}}{\partial t}>$ and employ one of the BO conditions $<u_{i}, u_{j}>=\lambda_{j} \delta_{i j}$ and the evolution equation for $\bar{u}$ to obtain

$$
\lambda_{j} \frac{d Y_{j}(t ; \omega)}{d t}=-\sum_{i=1}^{N} S_{j i} Y_{i}+<\mathcal{L}[u]-E[\mathcal{L}[u]], u_{j}>.
$$

Hence, the equation for $\mathbf{Y}$ will take the final form (12b). The fact that $S$ is equivalent to (13c) will be proved later. 
We multiply equation (14) with $Y_{j}$ and apply the mean value operator to get

$$
\frac{\partial \bar{u}}{\partial t} E\left[Y_{j}\right]+\sum_{i=1}^{N} E\left[\frac{d Y_{i}}{d t} Y_{j}\right] u_{i}+\sum_{i=1}^{N} E\left[Y_{i} Y_{j}\right] \frac{\partial u_{i}}{\partial t}=E\left[\mathcal{L}[u] Y_{j}\right]
$$

By defining the matrix $M$ whose entries are $M_{i j}=E\left[Y_{i} \frac{d Y_{j}}{d t}\right]$ and using the BO condition we have

$$
\frac{\partial u_{j}}{\partial t}=-\sum_{i=1}^{N} M_{j i} u_{i}+p_{j}
$$

which is exactly the evolution equation (12c).

Now we prove that the two matrices $S$ and $M$ defined as the above are the same as those given in equations (13c) and (13b), respectively. Indeed, by multiplying $Y_{k}$ on the both sides in equation (12b) and then taking the expectation we get

$$
\lambda_{j} M_{k j}=-\sum_{i=1}^{N} S_{j i} E\left[Y_{i} Y_{k}\right]+<E\left[\mathcal{L}[u] Y_{k}\right], u_{j}>
$$

where we use $E\left[(\mathcal{L}[u]-E[\mathcal{L}[u]]) Y_{k}\right]=E\left[\mathcal{L}[u] Y_{k}\right]$ because of the linearity of the expectation and $E\left[Y_{k}\right]=0$. By applying the $\mathrm{BO}$ condition we have

$$
\lambda_{j} M_{k j}=-S_{j k}+G_{j k}
$$

Interchanging the indices $k$ and $j$ yields

$$
\lambda_{k} M_{j k}=-S_{k j}+G_{k j}
$$

This holds for $k \neq j$. For $j=k$, we have $S_{j j}=G_{j j}$ since the diagonal entries of $M$ are zero. Summing up the last two equations and using skew-symmetric properties for $S$ for nondiagnoal elements and $M$ yield

$$
M_{j k}= \begin{cases}\frac{G_{j k}+G_{k j}}{-\lambda_{j}+\lambda_{k}}, & \text { if } j \neq k \\ 0, & \text { if } j=k\end{cases}
$$

and substituting it back into equation (16) we get the explicit form for $S$

$$
S_{j k}= \begin{cases}\frac{\lambda_{k}}{-\lambda_{j}+\lambda_{k}} G_{j k}+\frac{\lambda_{j}}{-\lambda_{j}+\lambda_{k}} G_{k j}, & \text { if } j \neq k \\ G_{j j}, & \text { if } j=k .\end{cases}
$$

This completes the proof. 
Remark 4. The evolution equations $(12 a)-(12 c)$ can be recasted into matrix form with $\mathbf{u}=$ $\left(u_{1}, \ldots, u_{N}\right), \mathbf{Y}=\left(Y_{1}, \ldots, Y_{N}\right)$ and $\Lambda=\operatorname{diag}\left(\lambda_{1}, \ldots, \lambda_{N}\right)$ as follows:

$$
\begin{aligned}
\frac{\partial \bar{u}(t, x)}{\partial t} & =E[\mathcal{L}[u]], \\
\frac{d \mathbf{Y}(t ; \omega)}{d t} \Lambda & =-\mathbf{Y} S^{T}+\mathbf{h}, \\
\frac{\partial \mathbf{u}(t, x)}{\partial t} & =\mathbf{u} M+\mathbf{p},
\end{aligned}
$$

where $S^{T}$ is the transpose of the matrix $S$.

We note that the rate of change of the basis and stochastic coefficients is associated with the matrix $G$ whose entries are $G_{i j}=<E\left[\mathcal{L}[u] Y_{j}\right], u_{i}>$, and the matrix $S$ and $M$ have closed form. The denominator in nondiagonal entries of the matrix $M$ in Equation (13b) can be zero if two eigenvalues become identical. However, according to [1], the eigenvalues do not cross out and this would not be the case.

The diagonal entries for $S$ account for how the eigenvalues change in time $S_{i i}=\frac{1}{2} \frac{d \lambda_{i}(t)}{d t}$ as discussed in Remark 3, which can be computed exactly by equation (13c). This can be used as a measure of criterion of when to add or remove modes; if the lowest eigenvalue grows quickly and is larger than certain value, a new mode needs to be added.

Remark 5. Both DO and BO representations can be viewed as an extension of Karhunen-Loeve representation in time under different assumptions. It is shown in Section 4 that they are equivalent through the matrix differential equation; in other words, there is an one-to-one mapping between the BO components and DO components. However, we have observed that BO is numerically more stable than DO, in particular for high modes in non-linear problems.

\section{Equivalence of $\mathrm{BO}$ and $\mathrm{DO}$}

We show that the $\mathrm{BO}$ and $\mathrm{DO}$ are equivalent in the sense that the $\mathrm{DO}$ components can be derived from the $\mathrm{BO}$ components via the invertible matrix that evolves through the matrix ordinary differential equation, and vice versa. Let $U=\left(u_{1}, u_{2}, \ldots, u_{N}\right)^{T}, \tilde{U}=\left(\tilde{u}_{1}, \tilde{u}_{2}, \ldots, \tilde{u}_{N}\right)^{T}, Y=\left(Y_{1}, Y_{2}, \ldots, Y_{N}\right)^{T}$ and $\tilde{Y}=\left(\tilde{Y}_{1}, \tilde{Y}_{2}, \ldots, \tilde{Y}_{N}\right)^{T}$ be. Consider the linear transformation

$$
\begin{aligned}
Y & =\Lambda^{-\frac{1}{2}} P \tilde{Y} \\
U & =\Lambda^{\frac{1}{2}} P \tilde{U}
\end{aligned}
$$

where $\Lambda=\operatorname{diag}\left(\lambda_{1}, \ldots, \lambda_{N}\right)$ with $\lambda_{i}, i=1, \ldots, N$ being the positive eigenvalues of the covariance operator in equation (9) and $P$ satisfies the matrix differential equation

$$
\begin{aligned}
& \dot{P}=-\Lambda^{-\frac{1}{2}} \Sigma \Lambda^{-\frac{1}{2}} P, \\
& P(0)=I_{N} \text {, }
\end{aligned}
$$


where $\dot{P}$ is the time derivative of $P, I_{N}$ is the $N \times N$ identity matrix and $\Sigma$ is the skew-symmetric part of the matrix $S$ in equation (13c), i.e. $\Sigma_{i j}=S_{i j}$ for $i \neq j$ and $\Sigma_{i i}=0$ for $i=1, \ldots, N$.

Remark 6. The solution $P(t)$ to the matrix differential equation (22) remains the orthogonal matrix for every time $t \geq 0$ given the initial condition $P(0)$ be an orthogonal matrix. Indeed the coefficient $F(t) \equiv-\Lambda^{-\frac{1}{2} \Sigma} \Lambda^{-\frac{1}{2}}$ of $P$ in equation (22) is skew-symmetric because $\Sigma$ is skew-symmetric, and then we have

$$
\begin{aligned}
\frac{d}{d t} P^{T}(t) P(t) & =\dot{P}^{T}(t) P(t)+P^{T}(t) \dot{P}^{T}(t) \\
& =(F P)^{T} P+P^{T} F P \\
& =P^{T}\left(F^{T}+F\right) P \\
& =O, \quad t \geq 0 .
\end{aligned}
$$

Therefore $P^{T}(t) P(t)=P^{T}(0) P(0)=I, t \geq 0$.

We are now ready to establish the connection between the BO and the DO components.

Theorem 3. Suppose that $Y, U, \tilde{Y}$, and $\tilde{U}$ satisfy equations (21a)-(21b). Then, there exists the unique transformation between the $B O$ and DO components: $Y$ and $U$ are the solutions to the $B O$ evolution equations (12a)-(12c) if and only if $\tilde{Y}$ and $\tilde{U}$ are the solutions to the DO evolution equations (5a)-(5c).

Proof. Assume that $Y$ and $U$ are the solutions to the BO evolution equations (12a)-(12c). Then we will prove that $\tilde{Y}$ and $\tilde{U}$ are the solutions to the DO evolution equations (5a)-(5c) by showing the following three properties: i) $\tilde{U}$ is an orthonormal basis, ii) $Y^{T} U=\tilde{Y}^{T} \tilde{U}$, and iii) $\tilde{U}$ satisfy the DO condition and $(\tilde{U}, \tilde{Y})$ are DO components.

First, define the inner product of matrix $U U^{T}$ in the physical space by $<U U^{T}>$ whose $(i, j)$-th entry is $\left\langle u_{i}, u_{j}\right\rangle$. According to the $\mathrm{BO}$ assumption on the basis $U$, we have

$$
\begin{aligned}
\Lambda & =<U U^{T}> \\
& =<\Lambda^{\frac{1}{2}} P \tilde{U}\left(\Lambda^{\frac{1}{2}} P \tilde{U}\right)^{T} \\
& =\Lambda^{\frac{1}{2}} P<\tilde{U} \tilde{U}^{T}>P^{T} \Lambda^{\frac{1}{2}} .
\end{aligned}
$$

Multiplying $P^{T} \Lambda^{-\frac{1}{2}}$ and $\Lambda^{-\frac{1}{2}} P$ to the left and right, respectively on the both sides yields

$$
\begin{aligned}
<\tilde{U} \tilde{U}^{T}> & =P^{T} \Lambda^{-\frac{1}{2}} \Lambda \Lambda^{-\frac{1}{2}} P \\
& =P^{T} P \\
& =I
\end{aligned}
$$

where we used the property of orthogonal matrix $P$. Hence $\tilde{U}$ is an orthonormal basis. 
Second, the BO and DO representation to the solution $u(x, t ; \omega)$ has the same form:

$$
\begin{aligned}
u(x, t ; \omega) & =\bar{u}(x, t)+\sum_{i=1}^{N} u_{i}(x, t) Y_{i}(t ; \omega) \\
& =\bar{u}(x, t)+\sum_{i=1}^{N} \tilde{u}_{i}(x, t) \tilde{Y}_{i}(t ; \omega),
\end{aligned}
$$

where $\left(u_{i}, Y_{i}\right)_{i=1}^{N}$ and $\left(\tilde{u}_{i}, \tilde{Y}_{i}\right)_{i=1}^{N}$ are the $\mathrm{BO}$ and DO components, respectively. Indeed, we obtain this directly using equations (21a)-(21b)

$$
U^{T} Y=\left(\Lambda^{\frac{1}{2}} P \tilde{U}\right)^{T} \Lambda^{-\frac{1}{2}} P \tilde{Y}=\tilde{U}^{T} P^{T} \Lambda^{\frac{1}{2}} \Lambda^{-\frac{1}{2}} P \tilde{Y}=\tilde{U}^{T} P^{T} P \tilde{Y}=\tilde{U}^{T} \tilde{Y}
$$

Finally, we have by the definition of the transformation

$$
U=\Lambda^{\frac{1}{2}} P \tilde{U}
$$

from which we have

$$
\begin{aligned}
\dot{U} & =\frac{1}{2} \dot{\Lambda} \Lambda^{-\frac{1}{2}} P \tilde{U}+\Lambda^{\frac{1}{2}} \dot{P} \tilde{U}+\Lambda^{\frac{1}{2}} P \dot{\tilde{U}} \\
& =(S-2 \Sigma) \Lambda^{-\frac{1}{2}} P \tilde{U}+\Lambda^{\frac{1}{2}} P \dot{\tilde{U}}
\end{aligned}
$$

where the second equality comes from the equation (22) and $S=\Sigma+\frac{1}{2} \dot{\Lambda}$. We have by the definition of the matrix $S$ as in equation (10)

$$
S=<U \dot{U}^{T}>
$$

and putting the above two equations for $U$ and $\dot{U}$ all together yields

$$
\begin{aligned}
S & =<\Lambda^{\frac{1}{2}} P \tilde{U}\left((S-2 \Sigma) \Lambda^{-\frac{1}{2}} P \tilde{U}+\Lambda^{\frac{1}{2}} P \dot{\tilde{U}}\right)^{T} \\
& =<\Lambda^{\frac{1}{2}} P \tilde{U} \tilde{U}^{T} P^{T} \Lambda^{-\frac{1}{2}}(S-2 \Sigma)^{T}>+<\Lambda^{\frac{1}{2}} P \tilde{U} \dot{\tilde{U}}^{T} P^{T} \Lambda^{\frac{1}{2}}> \\
& =(S-2 \Sigma)^{T}+\Lambda^{\frac{1}{2}} P<\tilde{U} \dot{\tilde{U}}^{T}>P^{T} \Lambda^{\frac{1}{2}}
\end{aligned}
$$

where we employed $P P^{T}=I,<\tilde{U} \tilde{U}^{T}>=I$ to get the third equality. Hence we have

$$
\begin{aligned}
\frac{1}{2} \Lambda^{\frac{1}{2}} P<\tilde{U} \dot{\tilde{U}}^{T}>P^{T} \Lambda^{\frac{1}{2}} & =\frac{S-S^{T}}{2}-\Sigma \\
& =O
\end{aligned}
$$

because $\Sigma$ is the skew-symmetric part of the matrix $S$ that is exactly the first term on the right hand side and we obtain $<\tilde{U} \dot{\tilde{U}}^{T}>=O$ that is precisely the DO condition in vector notation. This completes the proof that $\tilde{Y}$ and $\tilde{U}$ are the solutions to the DO evolution equations.

The same procedure can be used to prove that if $\tilde{Y}$ and $\tilde{U}$ are the solutions to the DO evolution equations, then $Y$ and $U$ are the solutions to the BO evolution equations. 
The $\mathrm{BO}$ and $\mathrm{DO}$ representation come from the KL decomposition and require that both basis and stochastic coefficients are time-dependent. Hence there exists some redundancy in the representation. In order to remove redundancy different constraints are imposed; DO require that the evolution of the basis is normal to the space $V_{S}$ spanned by the basis called the $D O$ condition while $\mathrm{BO}$ requires that both the basis and stochastic coefficients are orthogonal with respect to underying measure called the $B O$ condition. Theorem 3 implies that both methods are equivalent in the sense that one can be derived from the other, and vice versa through the orthogonal matrix as in equations (21a)-(21b). Indeed, if equations (21a)-(21b) are plugged into the BO evolution equations (12a)-(12c), then the DO evolution equations (5a)-(5c) are obtained, and vice versa.

\section{Numerical example: Burgers equation}

In this section, we consider the Burgers equation with random forcing and demonstrate convergence with respect to the number of DO modes.

Consider the following stochastic Burgers equation with random forcing

$$
\begin{aligned}
\frac{\partial u}{\partial t}+u \frac{\partial u}{\partial x} & =\nu \frac{\partial^{2} u}{\partial x^{2}}+\frac{1+\xi}{2} \sin (2 \pi t), \quad \forall(t, x) \in[0, T] \times D=[0,2 \pi] \\
u(0, x) & =g(x), \quad \forall x \in D
\end{aligned}
$$

where $\xi \sim U[-1,1]$ and the initial condition $g(x)$ is given as

$$
g(x)=0.5(\exp (\cos (x))-1.5) \sin (x+2 \pi \cdot 0.37) .
$$

We take $\nu=0.05$. Note that the period of the forcing is one. Using the DO representation, we obtain the form of the evolution operator $\mathcal{L}$ and some necessary forms:

$$
\begin{aligned}
\mathcal{L}[u(x, t ; \omega)]= & -u u_{x}+\nu u_{x x}+\frac{1+\xi}{2} \sin (2 \pi t) \\
= & -\bar{u} \bar{u}_{x}-Y_{i} \frac{\partial}{\partial x}\left(u_{i} \bar{u}\right)-Y_{i} Y_{j} u_{i} \frac{\partial u_{j}}{\partial x}+\nu\left(\bar{u}_{x x}+Y_{i} \frac{\partial^{2} u_{i}}{\partial x^{2}}\right)+\frac{1+\xi}{2} \sin (2 \pi t) \\
E[\mathcal{L}(u)]= & -\bar{u} \bar{u}_{x}-C_{i j} u_{i} \frac{\partial u_{j}}{\partial x}+\nu \bar{u}_{x x}+0.5 \sin (2 \pi t) \\
E\left[\mathcal{L}(u) Y_{j}\right]= & -\left(C_{i j} u_{i} \bar{u}_{x}+C_{k j} \frac{\partial u_{k}}{\partial x} \bar{u}+C_{i k j} u_{i} \frac{\partial u_{k}}{\partial x}\right) \\
& +\nu C_{i j} \frac{\partial^{2} u_{i}}{\partial x^{2}}+E\left[\frac{\xi}{2} Y_{j}\right] \sin (2 \pi t),
\end{aligned}
$$

where $C_{i j k}=E\left[Y_{i} Y_{j} Y_{k}\right]$. Note that $E\left[\mathcal{L} Y_{j}\right]$ involves the third moment of the stochastic coefficients and hence the PDE for $u_{i}$ is more complicated than the one in the advection equation in the previous section. Since the initial condition is deterministic as in the advection equation, the $Y_{i}, i=1, \ldots, N$ 
at the initial time become zero, which makes the covariance matrix for $Y_{i}$ singular. Hence, we use the hybrid method to avoid the singularity due to the deterministic initial condition.

Unlike the advection problem where only one time step is enough to switch from PC to DO or $\mathrm{BO}$, we need to march for more time steps to allow the stochasticity of the system to develop fully. We have performed some sensitivity studies to see how to choose the switching time from PC to DO but a more systematic future study is required. We can choose the number of modes at the switching time based on the eigenvalues of $C_{u\left(\cdot, t_{s}\right)}(x, y)$. One criterion is to choose the number of modes such that the sum of corresponding eigenvalues makes up to more than a threshold, say $99 \%$ of the total.

The eigenvalues of KL decomposition of the solution at ten different times are shown in Figure 1. Note that the eigenvalues of KL decomposition are the same as those of the covariance matrix $C$ whose $(i, j)$ index is $E\left[Y_{i} Y_{j}\right]$. We choose the switching time to be $t_{s}=1.0$ and the number of DO or BO modes to be 6 based on Figure 1. Note that the number of modes should be increased to capture the same percentage of the energy as the system evolves in time.
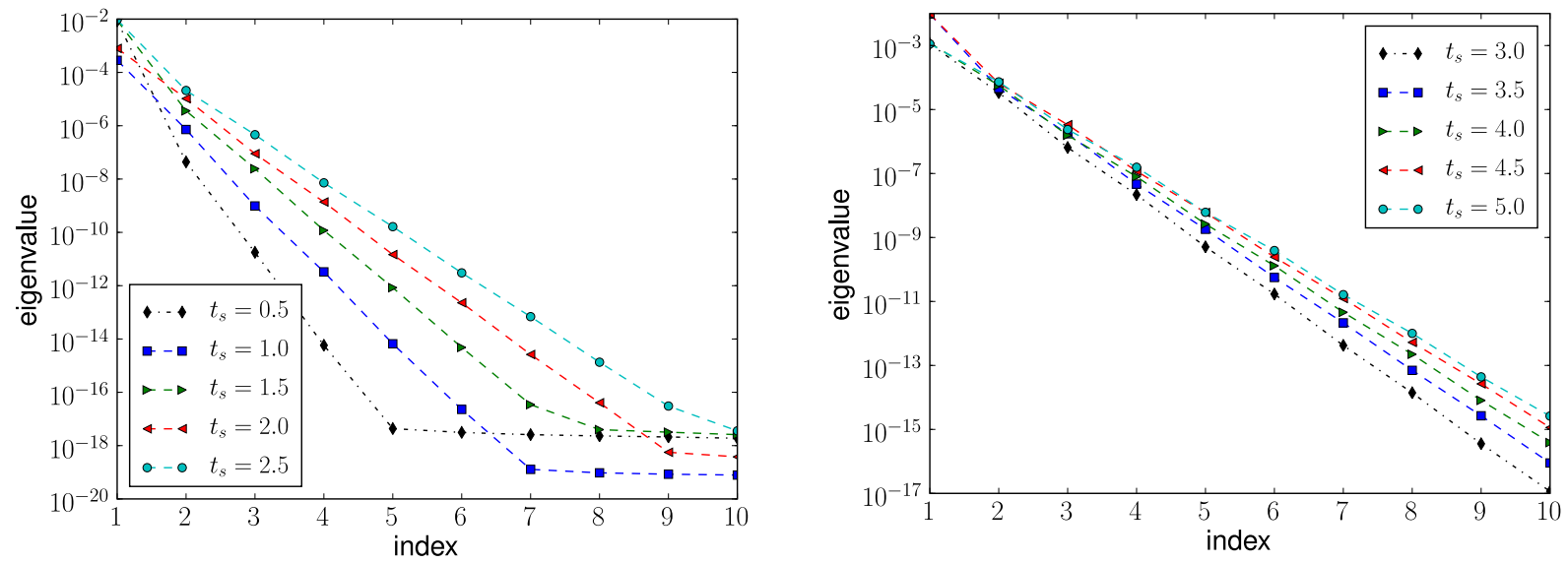

Figure 1: The eigenvalues of the covariance matrix $C$ whose $(i, j)$ index is $E\left[Y_{i} Y_{j}\right]$ at different switch times $t_{s}$. Left: eigenvalues for $t_{s}=0.5 j, j=1, \ldots, 5$, Right: eigenvalues for $t_{s}=0.5 j, j=6, \ldots, 10$. The parameters used are $\nu=0.05, N_{s}=128, N_{r}=64$ and $\Delta t=0.001$.

The parameters are as follows:

$$
\Delta t=0.001, t_{s}=1, t_{f}=5, N_{s}=128, N_{r}=64, N=6 .
$$

We choose $N=6$ because, at $t_{s}=1$, the sixth mode is the largest eigenmode whose eigenvalue is larger than the threshold value. Fourier collocation in the physical space and Legendre-Gauss collocation in the parametric space are used for discretization. The third-order Adams-Bashforth (AB3) is used as a time integrator to minimize the error due to the time discretization. 
The mean and variance at $t=5$ using the hybrid method are shown in Figure 2; good agreement with the exact solution is achieved. The $L_{2}$ error for the mean and variance are shown in Figure 3 where BO has better accuracy than DO by one order of magnitude. DO and BO are tested with different number of modes up to 6 . They have the same accuracy for the first four modes but BO is better than DO for higher modes. While they are equivalent as shown in Appendix this suggests that BO gives numerically more stable scheme than DO as shown in Figure 4; the DO evolution equation for the basis needs an inverse of matrix whose condition number for higher number is large that may affect numerical instability. Further research is required in order to document this point.

Figure 4 shows the exponential convergence obtained with respect to the number of modes at time $t=5$. As mentioned above both $\mathrm{DO}$ and $\mathrm{BO}$ have the same accuracy with lower modes but $\mathrm{BO}$ is more accurate than DO with higher modes 5 and 6 . This example is the first demonstration of the fast convergence of the DO or BO method for a nonlinear SPDE.

\section{References}

[1] T. Sapsis, P. Lermusiaux, Dynamically orthogonal field equations for continuous stochastic dynamical systems, Physica D 238 (2009) 2347-2360.

[2] M. Cheng, T. Y. Hou, Z. Zhang, A dynamic bi-orthogonal stochastic method for solving timedependent stochastic pdes, in: Workshop on Uncertainty Quantification at ICERM, 2012.

[3] K. Sobczyk, Stochastic Wave Propagation, Elsevier Publishing Company, 1985.

[4] Y. Rozanov, Random Fields and Stochastic Partial Differential Equations, Kluwer Academic Press, 1996.

[5] M. Gerritsma, J.-B. van der Steen, P. Vos, G. E. Karniadakis, Time-dependent generalized polynomial chaos, J. Comput. Phys. 229 (2010) 8333-8363.

[6] J. Foo, X. Wan, G. E. Karniadakis, The multi-element probabilistic collocation method: error analysis and simulation, J. Comput. Phys. 227 (2008) 9572-9595.

\section{Publications from this Award}

1. H. Baek and G.E. Karniadakis, "Sub-iteration leads to accuracy and stability enhancements of semi-implicit schemes for the Navier-Stokes equations", J. Comp. Phys., vol. 230, pp. 4384-4402, 2011.

2. X. Yang, D. Venturi, C. Chen, C. Chryssotomidis, G.E. Karniadakis, "POD-based constrained sensor placement and field reconstruction from noisy ocean measurements: Application to Nantucket Sound", J. of Geophysical Research Oceans, vol. 115, C12072, 2010. 

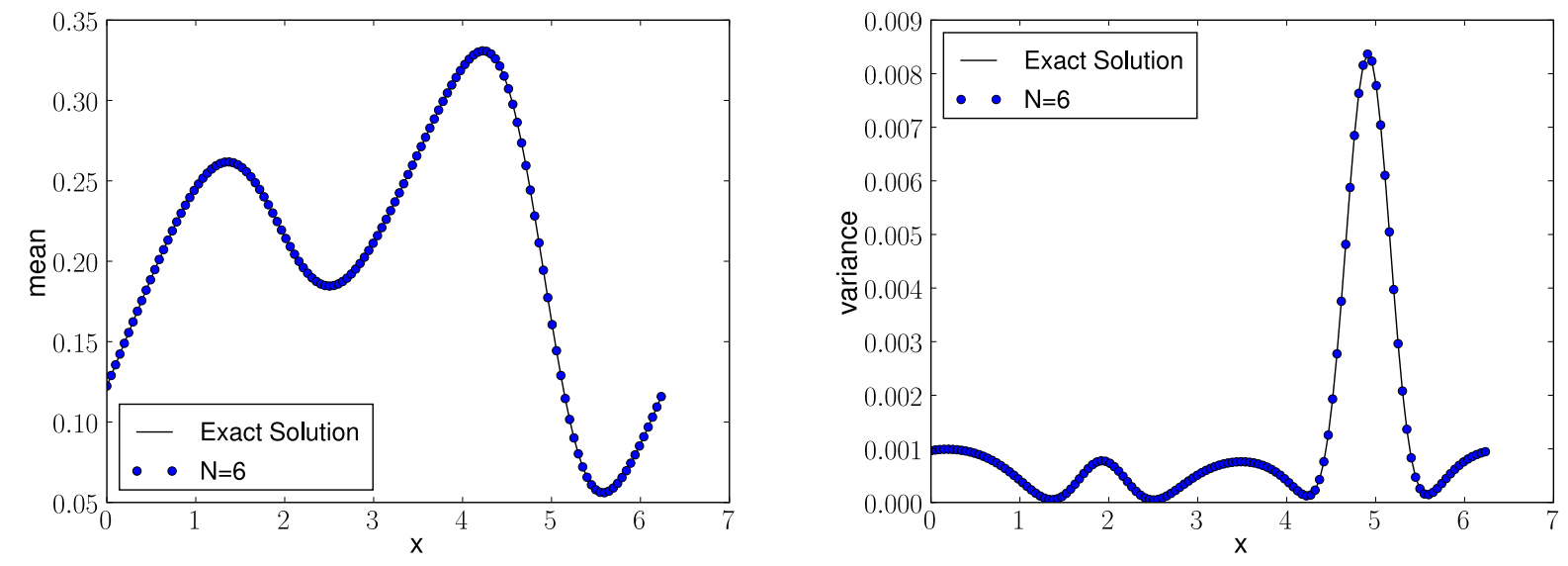

Figure 2: Mean (left) and variance (right) of the solution at $t=5$ for the Burgers equation. The switching time $t_{s}$ is 1 and the number of DO modes is 6 . The mean and variance from the probabilistic collocation method with $N_{r}=512$ using the fourth-order Runge-Kutta method are considered to be the exact solution.
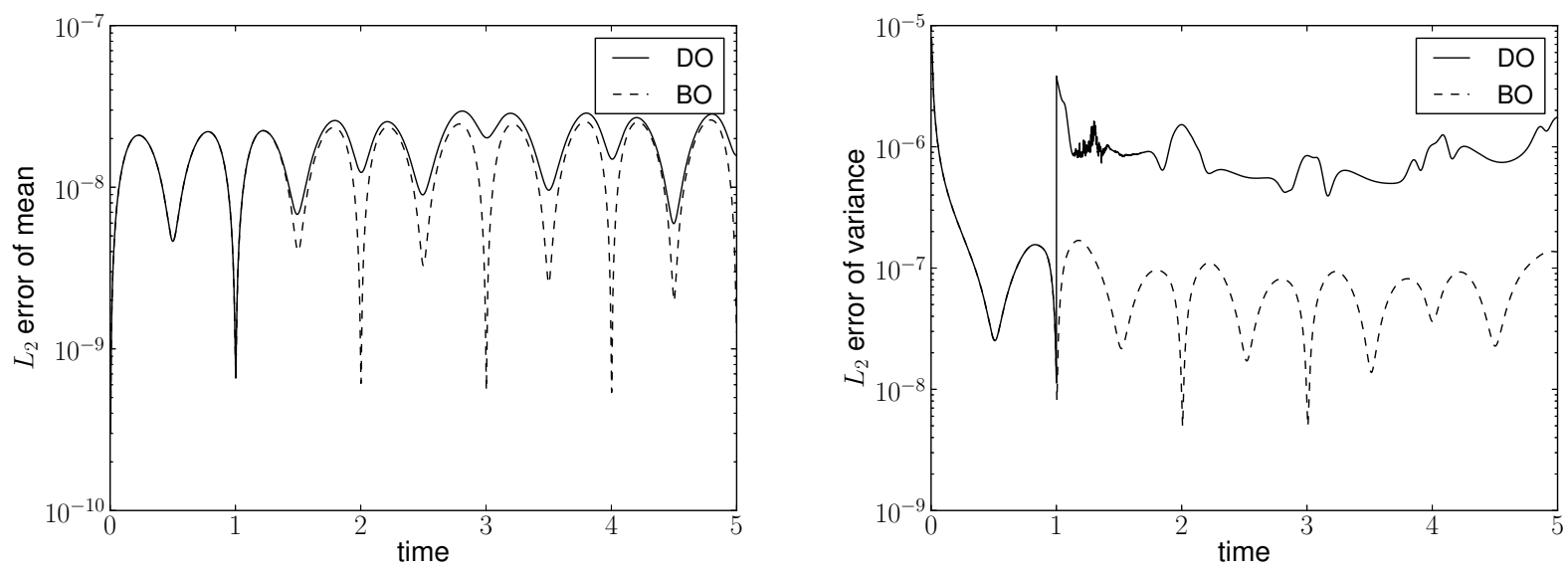

Figure 3: Relative $L_{2}$ error for the mean (left) and variance (right) of the solution for the Burgers equation with random forcing using DO and BO. Both methods have the same accuracy for the mean while $\mathrm{BO}$ is an order of magnitude more accurate compared to $\mathrm{DO}$ for the variance. $\mathrm{BO}$ is numerically more stable than DO for high modes while they have the same accuracy for low modes. Note that the switching time is 1 and the error before the switching time is the same as collocation method is used in the hybrid method. 


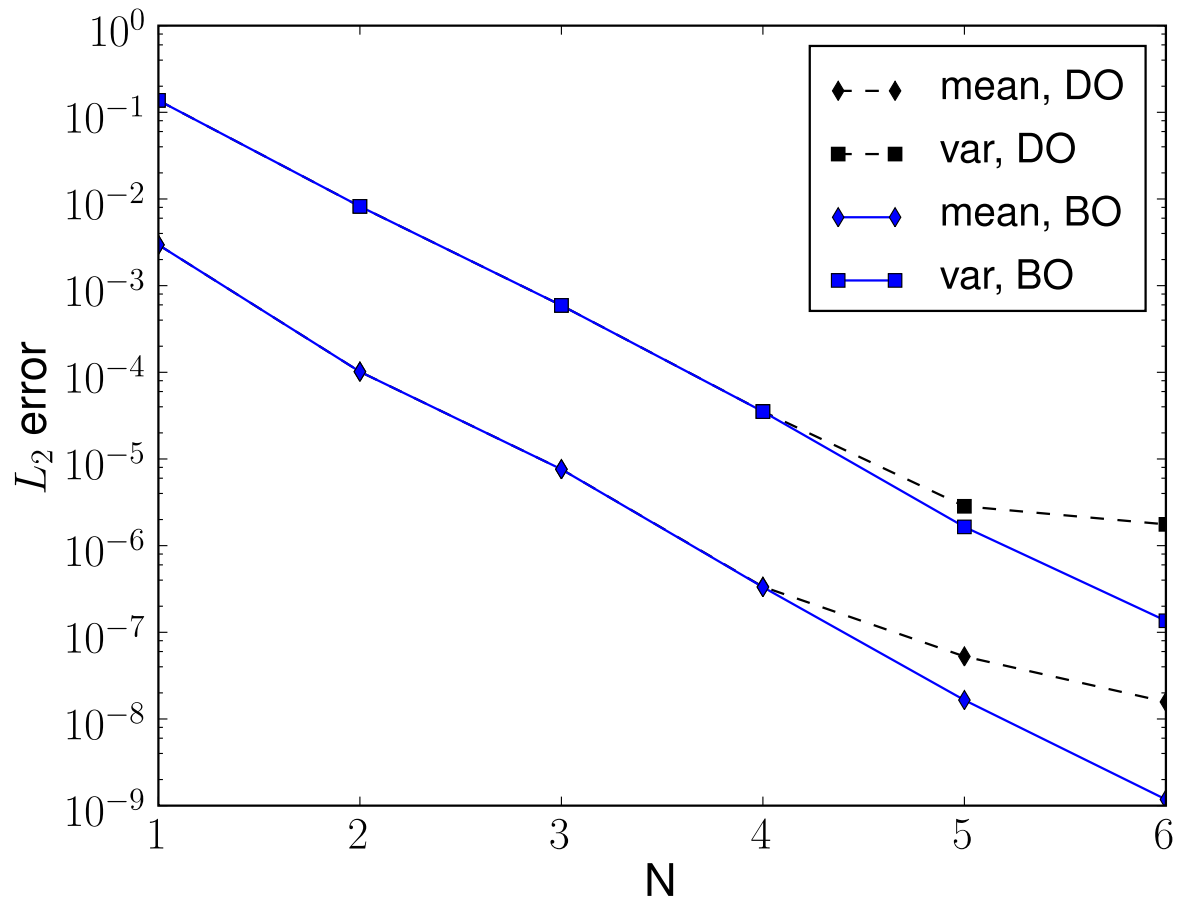

Figure 4: Relative $L_{2}$ error for the mean and variance at $t=5$. Exponential convergence is observed as the number of modes increases. They have the same accuracy through $N=4$ but BO is better than DO for high modes. 
3. Z. Zhang, M. Choi, G.E. Karniadakis, "Error estimates for the ANOVA method with polynomial chaos interpolation: Tensor product functions, SIAM J. Sci. Comp., vol. 34, A1165A1186, 2012.

4. X. Yang, M. Choi, G. Lin, G.E. Karniadakis, "Adaptive ANOVA decomposition of stochastic incompressible and compressible flows", J. Comp. Phys., vol. 231, pp. 1587-1614.

5. M. Choi, T. Sapsis, G.E. Karniadakis, "A convergence study of SPDEs using combined polynomial chaos and dynamically-orthogonal schemes", J. Comp. Phys., online, April 1, 2013.

\section{Personnel and Cost}

George Karniadakis (PI); Bruce Caswell (senior personnel); Daniele Venturi (postdoc, partial), Xiu Yang (PhD student, partial); Mohsen Zayemouri (PhD student, partial); Minsook Choi (PhD student, partial). 